\title{
APPLICATIONS OF THE SPACES OF HOMOGENEOUS POLYNOMIALS TO SOME PROBLEMS ON THE BALL ALGEBRA
}

\author{
J. BOURGAIN
}

\begin{abstract}
Denote by $B_{2}$ the unit ball in $C^{2}$. The existence is shown of a uniformly bounded orthonormal basis in $H^{2}\left(B_{2}\right)$, by constructing such systems in the spaces of homogeneous polynomials. In the second part of the paper, those spaces of homogeneous polynomials are exploited to disprove the existence of generalized analytic projections, the so-called $\left(i_{p}-\pi_{p}\right)$ property, for the ball algebra.
\end{abstract}

Summary. In the first part of the paper we use a construction in the spaces of homogeneous polynomials on the 2-dimensional complex ball $B_{2}$ to generate an orthonormal basis for the space $H^{2}\left(B_{2}\right)$ which is uniformly bounded. The existence of such a system answers a question raised by $\mathrm{W}$. Rudin. ${ }^{1}$ The second part is devoted to the failure of the $\left(i_{p}-\pi_{p}\right)$ theorem, known for the disc algebra $A(D)$, in case of the ball algebras $A\left(B_{m}\right), m>1$. It is proved that the ideals of $p$-summing and $p$-integral operators $(p \neq 2)$ in $A\left(B_{2}\right)$ are distinct. This fact solves negatively a problem considered in [2].

1. Introduction and terminology. Denote by $\langle$,$\rangle the inner product on \mathbf{C}^{2}$, by $\boldsymbol{B}_{2}$ the closed unit ball of $\mathbf{C}^{2}$, and by $S_{2}=\partial B_{2}=\left\{\zeta \in \mathbf{C}^{2} ;\|\zeta\|=\langle\zeta, \zeta\rangle^{1 / 2}=1\right\}$ the unit sphere. Under the parametrization $\zeta=(z, w), z=\sqrt{\rho} e^{i \theta}$, and $w=\sqrt{1-\rho} e^{i \psi}$, the normalized Haar measure $\sigma$ on $S_{2}$ is given by $d \sigma=\left(1 / 4 \pi^{2}\right) d \rho d \theta d \psi(0 \leqslant \rho \leqslant 1$, $0 \leqslant \theta, \psi \leqslant 2 \pi)$. For $1 \leqslant p \leqslant \infty$, the spaces $L^{p}\left(S_{2}\right)=L^{p}\left(S_{2}, \sigma\right)$ are defined in the usual way. The ball algebra $A\left(B_{2}\right)$ consists of continuous functions in $B_{2}$ which are analytic in the interior of $B_{2}$ and identifies with a subspace of the space $C\left(S_{2}\right)$ of continuous functions on $S_{2}$ by restriction to the sphere. The spaces $H^{p}\left(B_{2}\right)(1 \leqslant p$ $<\infty$ ) are obtained as the closure of $A\left(B_{2}\right)$ in $L^{p}\left(S_{2}\right)$. They are generated by the polynomial spaces $\mathscr{P}_{N}=\operatorname{span}\left\{z^{j} w^{N-j} ; j=0,1, \ldots, N\right\}, N=0,1, \ldots$, where $\mathscr{P}_{N}$ consists of the homogeneous polynomials of degree $N$. A remarkable property is the fact that the orthogonal projection onto $\mathscr{P}_{N}$,

$$
P_{N} f=P f=c_{N}^{-1} \int\langle\eta, \zeta\rangle^{N} f(\zeta) d \sigma(\zeta), \quad c_{N}=\int|z|^{2 N} d \sigma
$$

Received by the editors December 23, 1983.

1980 Mathematics Subject Classification. Primary 46E15, 32A99; Secondary 42A56.

${ }^{1}$ During a seminar conference at Princeton University, October 1983. 
is bounded under the $L^{p}$-norm $(1 \leqslant p \leqslant \infty)$ independently of $N$. One has, indeed,

$$
\|P\|_{L^{\infty}\left(S_{2}\right) \rightarrow L^{\infty}\left(S_{2}\right)}=c_{N}^{-1} \int|z|^{N} d \sigma \leqslant c \quad \text { (= numerical constant). }
$$

For $\zeta, \eta \in S_{2}$, let $d(\zeta, \eta)=1-|\langle\zeta, \eta\rangle|$, which is the natural metric for the projective space $\mathbf{P}=\mathbf{P}^{1}$. Notice that the modulus of a homogeneous polynomial on $S_{2}$ can be seen as a function on $\mathbf{P}$. We mention [3] as a standard reference for function theory on complex balls.

If $X, Y$ are Banach spaces, $\mathrm{II}_{p}(X, Y)$ and $\mathrm{I}_{p}(X, Y)(1 \leqslant p<\infty)$ denote the ideals of $p$-summing, respectively strictly $p$-integral, operators from $X$ to $Y$. The respective norms are given by

$$
\begin{aligned}
& \pi_{p}(u)=\sup \left\{\left.\left(\sum\left\|u\left(x_{i}\right)\right\|^{p}\right)^{1 / p}\left|\left\{x_{i}\right\} \subset X, \sum\right|\left\langle x_{i}, x^{*}\right\rangle\right|^{p} \leqslant 1\right. \\
& \left.\qquad \text { for } x^{*} \in X^{*},\left\|x^{*}\right\| \leqslant 1\right\}
\end{aligned}
$$

and

$$
i_{p}(u)=\inf \|A\|\|B\|,
$$

where this infimum is taken over all factorizations $u=B \circ I \circ A, I: L^{\infty}(\Omega, \mu) \rightarrow$ $L^{p}(\Omega, \mu)$ is the identity map, $A: X \rightarrow L^{\infty}(\mu)$, and $B: L^{p}(\mu) \rightarrow Y$.

It is well known that $\pi_{p}(u) \leqslant i_{p}(u)$ and $i_{p}(u) \leqslant c p^{2} \pi_{p}(u) /(p-1)(1<p<\infty$, $c=$ numerical) whenever $u$ is a $p$-summing operator on the disc algebra $A(D)$ (the reader will find details on this subject in [2]). The problem of whether or not this so-called $\left(i_{p}-\pi_{p}\right)$ property holds for ball and polydisc algebras was considered in the last section of [2]. The answer to this question turns out to be negative in both cases. The polydisc case was settled in [1] and we present here the argument for the ball algebra $A\left(B_{2}\right)$, relying on the spaces $\mathscr{P}_{N}$ defined above. The argument in the general case $A\left(B_{m}\right)(m \geqslant 2)$ is completely similar.

2. Construction of a uniformly bounded orthonormal basis. Since the spaces $\mathscr{P}_{N}$ are mutually orthogonal in $H^{2}\left(B_{2}\right)$, it will suffice to construct the basis in each of the spaces $\mathscr{P}_{N}$ with a uniform bound on the $L^{\infty}$-norm of the systems. Our approach is completely explicit. It is clear that the polynomials

$$
\zeta_{k}=(N+1)^{-1 / 2} \sum_{j=0}^{N} \sigma_{j} e^{2 \pi i j k / N+1} \frac{z^{j} w^{N-j}}{\left\|z^{j} w^{N-j}\right\|_{2}} \quad(k=0,1, \ldots, N)
$$

form an orthonormal basis for $\mathscr{P}_{N}$, whatever the choice of the signs $\sigma_{j}= \pm 1$. We claim that if $\left\{\boldsymbol{\sigma}_{j}\right\}_{j=0}^{N}$ is a transform of Rudin-Shapiro type (that is, coefficients of a Rudin-Shapiro polynomial [4, p. 33 for definition]) or any $N+1$ consecutive coefficients in some Rudin-Shapiro transform, then $\left\|\zeta_{k}\right\|_{\infty} \leqslant c(0 \leqslant k \leqslant N)$, where the constant $c$ is independent of the degree $N$. Replacing $z$ by $z \cdot e^{2 \pi i k / N+1}$ and using the parametrization $z=\sqrt{\rho} e^{i \theta}, w=\sqrt{1-\rho} e^{i \psi}$, the problem clearly reduces to an estimation of

$$
\sup _{\substack{0 \leqslant \rho \leqslant 1 \\ 0 \leqslant \theta \leqslant 2 \pi}}(N+1)^{1 / 2}\left|\sum_{j=0}^{N} \sigma_{j}\left\|z^{j} w^{N-j}\right\|_{2}^{-1} \rho^{j / 2}(1-\rho)^{(N-j) / 2} e^{i j \theta}\right| .
$$


In the sequel $c$ denotes various numerical constants. We make use of the following fact.

LEMMA 1. For $\left\{\sigma_{j}\right\}_{j=0}^{N}$ an interval of length $N+1$ in a Rudin-Shapiro transform, $0 \leqslant a<b \leqslant N$, and $\left(\lambda_{j}\right)_{a \leqslant j \leqslant b}$ a monotone sequence,

$$
\left\|\sum_{j=a}^{b} \lambda_{j} \sigma_{j} e^{i j \theta}\right\|_{L_{\infty}(T)} \leqslant c \sup \left|\lambda_{j}\right| \cdot(b-a)^{1 / 2} .
$$

Proof. By an argument of partial summation, we may, of course, take $\lambda_{j}=1$ and verify the majorization of $\left\|\sum_{j=a}^{b} \alpha_{j} e^{i j \theta}\right\|_{L^{\infty}(T)}$. If $[a, b]$ is a dyadic interval in a Rudin-Shapiro transform, $\left\{\sigma_{j}\right\}_{j=a}^{b}$ is in fact again such a transform, as a consequence of their construction. Hence, $\left\|\sum_{j=a}^{b} \sigma_{j} e^{i j \theta}\right\|_{L^{\infty}(T)} \leqslant 2(b-a)^{1 / 2}$ in this case. For a general interval we divide $[a, b]$ into dyadic intervals of decreasing length, and apply the previous estimate and the triangle inequality to get the result. Details are elementary and left to the reader.

To obtain a bound on (2) is a straightforward computation. Using the evaluation

$$
\left\|z^{j} w^{N-j}\right\|_{2}^{2}=\int_{0}^{1} \rho^{j}(1-\rho)^{N-j} d \rho=\frac{j !(N-j) !}{(N+1) !}
$$

and Stirling's formula, we see that for fixed $\rho, 0 \leqslant \rho \leqslant 1$,

$$
\begin{aligned}
\alpha_{j} & \stackrel{\text { def }}{=}(N+1)^{-1 / 2}\left\|z^{j} w^{N-J}\right\|_{2}^{-1} \rho^{j / 2}(1-\rho)^{N-j / 2} \\
& =c(N+1)^{-1 / 2}\left\{\int_{0}^{1}\left(\frac{u}{\rho}\right)^{j}\left(\frac{1-u}{1-\rho}\right)^{N-j} d u\right\}^{-1 / 2}
\end{aligned}
$$

is dominated by an expression of the form

$$
C \exp \left[-c(j-\rho N)^{2} / N \rho(1-\rho)\right] \min (j, N-j)^{-1 / 4} .
$$

Moreover, taking a derivative with respect to $j$ in the right member of (3), we verify that $\left\{\alpha_{j}\right\}_{j=0}^{N}$ increases until $j=j_{0} \sim \rho N$; then it decreases. We suppose $0 \leqslant \rho \leqslant 1 / 2$, the other case being symmetric.

Rewrite (2) as

$$
\left\|\sum_{j=0}^{N} \sigma_{j} \alpha_{j} e^{i j \theta}\right\|_{L_{\infty}(T)},
$$

which we estimate by applying Lemma 1 and (4). Write

$$
\left\|\sum_{j=0}^{N} \sigma_{j} \alpha_{j} e^{i j \theta}\right\|_{L_{\infty}(T)} \leqslant\left\|\sum_{j=0}^{j_{0}} \sigma_{j} \alpha_{j} e^{i j \theta}\right\|_{\infty}+\left\|\sum_{j=j_{0}+1}^{N} \sigma_{j} \alpha_{j} e^{i j \theta}\right\|_{\infty},
$$

where thus $\left\{\boldsymbol{\alpha}_{j}\right\}_{j=0}^{j_{0}}$ is increasing and $\left\{\boldsymbol{\alpha}_{j}\right\}_{j=j_{0}+1}^{N}$ decreasing. We handle the first sum and leave the details (essentially similar) for the second sum to the reader. Before using Lemma 1, we need a further splitting of $\sum_{j=0}^{j_{0}}$. Define inductively

$$
k_{0}=0, \quad k_{1}=1, \quad k_{r+1}=k_{r}+\left[k_{r}^{1 / 2}\right] \quad([]=\text { integer part }),
$$


and let $s$ be the largest integer for which $k_{s} \leqslant j_{0}$. By Lemma 1

$$
\begin{aligned}
\left\|\sum_{j=0}^{j_{0}} \boldsymbol{\sigma}_{j} \alpha_{j} e^{i j \theta}\right\|_{\infty} & \leqslant \sum_{r=0}^{s-1}\left\|\sum_{j=k_{r}}^{k_{r+1}-1} \sigma_{j} \alpha_{j} e^{i j \theta}\right\|_{\infty}+\left\|\sum_{j=k_{s}}^{j_{0}} \sigma_{j} \alpha_{j} e^{i j \theta}\right\|_{\infty} \\
& \leqslant c \sum_{r=0}^{s-1} \alpha_{k_{r+1}}\left(k_{r+1}-k_{r}\right)^{1 / 2}+c \alpha_{j_{0}}\left(j_{0}-k_{s}\right)^{1 / 2} \\
& \leqslant c \sum_{r=0}^{s-1} \alpha_{k_{r+1}} k_{r}^{1 / 4}+c \alpha_{j_{0}} j_{0}^{1 / 4}
\end{aligned}
$$

where, by (4),

$$
\alpha_{k_{r}} \leqslant c k_{r}^{-1 / 4} \exp \left[-c^{\prime}\left(j_{0}-k_{r}\right)^{2} / j_{0}\right] \text { and } \alpha_{j_{0}} \leqslant c .
$$

From the construction of the sequence $\left\{k_{r}\right\}_{r=0}^{s}$, it is clear that

$$
j_{0}-k_{r} \geqslant \frac{1}{4} j_{0}^{1 / 2} \min \left(s-r, j_{0}^{1 / 2}\right) \text {. }
$$

Hence, one ends up with the series $\sum_{r=0}^{s-1}\left[e^{-c(s-r)^{2}}+e^{-c j_{0}}\right]$, obviously converging.

REMARK. The construction of the $\zeta_{K}$ polynomials provide an explicit example of $N$ homogeneous polynomials $p$ for which $\|p\|_{2} \sim\|p\|_{\infty}$. They can be used to obtain singular positive RP-measures $\mu$ on $S_{2}$ and, hence, inner functions on the ball $B_{2}$ (see [3] for details). Denote by $K$ the Cauchy kernel of $B_{2}$ and define the operator $T$ acting on $C^{\infty}\left(S_{2}\right)$ into $C^{\infty}\left(S_{2}\right) \cap \operatorname{Re} A\left(B_{2}\right)$ by

$$
T f=2 \operatorname{Re} K[f]-\operatorname{Re} \int f .
$$

Clearly $T^{2}=T$. Define inductively

$$
f_{0}=1, \quad f_{j+1}=T\left[f_{j}\left(1+\operatorname{Re} p_{j+1}\right)\right]+\varepsilon_{j+1},
$$

where $f_{j+1}$ is a homogeneous polynomial of degree $N_{j+1}$ chosen large enough and $\left\|p_{j+1}\right\|_{\infty} \leqslant 1,\left\|p_{j+1}\right\|_{2}>\delta=$ constant. For $\varepsilon_{j+1}$ we take the smallest positive number to insure the positivity of $f_{j+1}$. It is not hard to see that the $N_{j}$ 's and $p_{j}$ 's can be chosen inductively such that $\sum \varepsilon_{j}<\infty$ and each $w^{*}$-limit point of the sequence $\left\{f_{j} d \sigma\right\}$ in $M(S)$ yields a singular measure. Let us give some further details. By taking $N_{j+1}=$ degree $\left(p_{j+1}\right)$ large enough, we can ensure that $f_{j+1}$ is an arbitrarily small perturbation of $f_{j}\left(1+\operatorname{Re} p_{j+1}\right)$ in the uniform norm. The point is that $f_{j} \cdot p_{j+1}$ is "almost" in $A(B)$. To ensure that $\mathrm{w}^{*}$-limit points of $\left\{f_{j} d \sigma\right\}$ are singular measures, we are led to force the Riesz product $\Pi\left(1+\operatorname{Re} p_{j+1}\right)$ to determine a singular measure. Assume $p_{1}, \ldots, p_{j}$ is obtained. After choice of $N=N_{j+1}$, consider the bounded orthonormal basis $\xi$ in $\mathscr{P}_{N}$, which we introduced above. Since $\xi$ is orthonormal, an invariance argument with respect to the unitary group acting on $S$ shows that

$$
\sum_{p \in \xi \cup(i \xi)}(\operatorname{Re} p(\zeta))^{2}=\sum_{p \in \xi}|p(\zeta)|^{2}=|\xi|=N+1
$$

pointwise for $\zeta \in S$. Since, for $|x| \leqslant 1$,

$$
(1+x)^{1 / 2} \leqslant 1+\frac{1}{2} x-c x^{2} \quad(c>0),
$$


we find by (1) (for $\left.\delta=\frac{1}{2}\|p\|_{\infty}^{-1}\right)$,

$$
\begin{aligned}
\frac{1}{2(N+1)} & \sum_{p \in \xi_{0}} \int f_{j}^{1 / 2}(1+\delta \operatorname{Re} p)^{1 / 2} \\
& \leqslant \int f_{j}^{1 / 2}+\frac{\delta}{4(N+1)} \sum_{p \in \xi_{0}} \int f_{j}^{1 / 2} \operatorname{Re} p-c^{\prime} \int f_{j}^{1 / 2},
\end{aligned}
$$

where $\xi_{0}=\xi \cup(i \xi)$. For $N$ large enough, the second term on the right side will essentially vanish. So, for some $p \in \xi \cup(i \xi)$ and $p_{j+1}=\delta p$, it follows that

$$
\int f_{j+1}^{1 / 2} d \sigma \leqslant(1-c) \int f_{j}^{1 / 2} d \sigma
$$

3. Absolutely summing operators. If $\mu$ is a positive Radon measure on $S_{2}$ and $1 \leqslant p<\infty$, define $H^{p}(\mu)$ as the closure of the space $A\left(B_{2}\right)$ in $L^{p}(\mu)$. The identity map $A(B) \rightarrow H^{p}(\mu)$ is a trivial example of a $p$-summing operator on the ball algebra. It turns out to be possible to find $\mu, d \mu / d \sigma=\Delta \in L^{1}(S)$, such that the latter operator is not $p$-integral whenever $p \neq 2$ and does not extend to $C(S)$ for $p>2$. The approach is virtually the same as the method described in [1] to disprove the $\left(i_{p}-\pi_{p}\right)$ property for the bidisc algebra $A\left(D^{2}\right)$. The following general lemma (see [1, Lemma 6.1]) will be used again.

LEMMA 2. Assume $X$ is a linear subspace of a $C(K)$-space ( $K$ compact) satisfying the $\left(i_{p}-\pi_{p}\right)$ property for a fixed $p, 1 \leqslant p<\infty$, i.e. the ratio $k_{p}(x)=\sup \left\{i_{p}(u) \mid u\right.$ is $a$ p-summing operator on $X$ with $\left.\pi_{p}(u) \leqslant 1\right\}$ is assumed finite. Further, let $\varepsilon>0$ and let $\left\{\phi_{j}\right\}_{j=1}^{n}$ be a finite sequence in the unit ball of $X$ such that the sets $\left[\left|\phi_{j}\right|>\varepsilon\right]=\{t \in$ $\left.K|| \phi_{j}(t) \mid>\varepsilon\right\}$ are mutually disjoint. Then there exists a decomposition in $X$, $\phi_{j}=\phi_{j}^{\prime}+\phi_{j}^{\prime \prime}$ with $\phi_{j}^{\prime}, \phi_{j}^{\prime \prime} \in X$, fulfilling the conditions

$$
\begin{gathered}
\left\|\phi_{j}^{\prime}\right\|_{\infty} \leqslant 3 k_{p}(X) n^{\max (2, p)^{-1} \varepsilon \quad(1 \leqslant j \leqslant n),} \\
\left\|\sum_{j=1}^{n}\left|\phi_{j}^{\prime \prime}\right|\right\|_{\infty} \leqslant 3 k_{p}(X) n^{\max \left(2, p^{\prime}\right)^{-1}} .
\end{gathered}
$$

Of course, here $K=S_{2}$, and $X=A\left(B_{2}\right)$, considered as a subspace of $C\left(S_{2}\right)$. Notice that if $\phi_{j} \in \mathscr{P}_{N_{j}}$ for some integer $N_{j}(1 \leqslant j \leqslant n)$ the existence of a decomposition for $\left\{\phi_{j}\right\}_{j=1}^{n}$ in $A(B)$ implies a decomposition with $\phi_{j}^{\prime}, \phi_{j}^{\prime \prime} \in \mathscr{P}_{N_{j}}$ again for each $j$. This fact is clear upon using the projection operators

$$
R_{N} f(\zeta)=\frac{1}{2 \pi} \int_{0}^{2 \pi} f\left(e^{i \theta} z, e^{i \theta} w\right) e^{-i N \theta} d \theta, \quad \zeta=(z, w)
$$

from $A(B)$ onto $\mathscr{P}_{N}$.

LEMMA 3. There exists a sequence $\left\{p_{j}\right\}_{j=1}^{n}$ of homogeneous polynomials on $B_{2}$, $d\left(p_{j}\right)=\operatorname{degree}\left(p_{j}\right)=N_{j}$, for which the sets $\left[\left|p_{j}\right|>\varepsilon\right]$ are disjoint and $\left\|\sum_{j=1}^{n}\left|q_{j}\right|\right\|_{\infty}>$ cne whenever $\left\{q_{j}\right\}_{j=1}^{n}$ are homogeneous polynomials, $d\left(q_{j}\right) \leqslant N_{j}$ and $\left\|p_{j}-q_{j}\right\|_{\infty}<c$ $(1 \leqslant j \leqslant n)$ (where $c>0$ is numerical). 
To see that this gives the desired result, take $\varepsilon=(c / 4) k_{p}(X)^{-1} n^{-\max (2, p)^{-1}}$ in Lemma 2. Assuming $k_{p}(X)<\infty$ for $X=A\left(B_{2}\right)$ gives, by Lemma $3\left(\phi_{j}^{\prime \prime}=q_{j}\right)$,

$$
\text { cne }<3 k_{p}(X) n^{\max \left(2, p^{\prime}\right)^{-1}}, \quad c k_{p}(X)^{2}>n^{1 / 2-\max \left(p, p^{\prime}\right)^{-1}},
$$

which leads to a contradiction by letting $n \rightarrow \infty$ if $p \neq 2$.

LEMMA 4. Let $1=(1,0)$ and $V_{\delta}=\left\{\zeta \in S_{2}|\langle\zeta, 1\rangle|>1-\delta\right\}$. Then the inequality

$$
\sup \left\{\mid f(\zeta) \| \zeta \in S \backslash V_{\delta}\right\} \geqslant c(1-\delta)^{N}|f(1)|
$$

holds whenever $f \in \mathscr{P}_{N}$.

Proof. For $f \in L^{\infty}(S)$ the inequality

$$
\|f\|_{L^{\infty}\left(\Upsilon \backslash V_{\delta}\right)} \geqslant c N(1-\delta)^{-N}\left|\int_{S} f(\eta)\langle l, \eta\rangle^{N} \chi_{\left[\left|\eta_{1}\right| \leqslant 1-\delta\right]} d \eta\right|
$$

clearly holds. On the other hand, for $f \in \mathscr{P}_{N}, f=P_{N} f$,

$$
f(\eta)=c_{N}^{-1} \int f(\zeta)\langle\eta, \zeta\rangle^{N} d \zeta, c_{N} \sim \frac{1}{N} .
$$

Substitution yields

$$
\|f\|_{L^{\infty}\left(S \backslash V_{\delta}\right)} \geqslant c N^{2}(1-\delta)^{-N}\left|\iint f(\zeta)\langle\eta, \zeta\rangle^{N}\langle 1, \eta\rangle^{N} \chi_{\left[\left|\eta_{1}\right| \leqslant 1-\delta\right]} d \eta d \zeta\right| .
$$

Performing the integration in $\eta$ gives

$$
\int\langle\eta, \zeta\rangle^{N}\langle 1, \eta\rangle^{N} \chi_{\left[\left|\eta_{1}\right| \leqslant 1-\delta\right]} d \eta=\alpha(1-\delta)^{2 N} \bar{\zeta}_{1}^{N} \quad \text { with } \alpha \sim \frac{1}{N} .
$$

So

$$
\|f\|_{L^{\infty}\left(S \backslash V_{\delta}\right)} \geqslant c N(1-\delta)^{N}\left|\int f(\zeta) \bar{\zeta}_{1}^{N}\right| \geqslant c^{\prime}(1-\delta)^{N}|f(1)| .
$$

The proof of Lemma 3 is now very much in the spirit of Lemma 6.3 in [1]. For $a \in S_{2}$ and $\alpha>0$, let $B(a, \alpha)=\left\{\zeta \in S_{2} \mid d(a, \zeta)<\alpha\right\}$, where $d(\zeta, \eta)=1-|\langle\zeta, \eta\rangle|$ is the distance in projective space. Since $\mathscr{P}_{N}$ and $d$ are invariant under the unitary group acting on $S_{2}$, Lemma 4 also implies

$$
\|f\|_{L^{\infty}(S \backslash B(a, \alpha))} \geqslant c(1-\alpha)^{N}|f(a)| \quad \text { if } f \in \mathscr{P}_{N} .
$$

Moreover, this inequality is, up to a constant, best possible, considering polynomial $f(\zeta)=\langle\zeta, a\rangle^{N}$.

Proof of Lemma 3. Fix $n$ and $\varepsilon>0$. We introduce inductively positive numbers and positive integers $1=\delta_{1}>\varepsilon_{1}>\delta_{2}>\varepsilon_{2}>\cdots>\delta_{j}>\varepsilon_{j}>\cdots>\delta_{n}>\varepsilon_{n}$ and $1=N_{1}<N_{2}<\cdots<N_{j}<\cdots<N_{n}$. For each $j, \xi_{j}$ will be a $\delta_{j}$-net in the set $R_{j}=S \backslash \cup_{s=1}^{j-1} \cup_{a \in \xi_{s}} B\left(a, \varepsilon_{s}\right)$, and we assume $B\left(a, \frac{1}{10} \delta_{j}\right) \subset R_{j}$ for all $a \in \xi_{j}$. We take $\xi_{1}=\{1\}$. The polynomials $\left\{p_{j}\right\}_{j=1}^{n}$ are given by

$$
p_{j}(\zeta)=\sum_{a \in \xi_{j}}\langle\zeta, a\rangle^{N_{j}}
$$

where $N_{j}$ is chosen large enough to imply $\left\|p_{j}\right\|_{\infty} \approx 1$ and

$$
\left[\left|p_{j}\right|>\varepsilon\right] \subset \bigcup_{a \in \xi_{j}} B\left(a, \varepsilon_{j}\right),
$$


where $\varepsilon_{j}$ is defined by $\left(1-\varepsilon_{j}\right)^{N_{j}}=\varepsilon / 2$. In the construction, $N_{j}$ depends on $\delta_{j}$. For $N_{j}$ sufficiently large, $\varepsilon_{j}$ becomes arbitrarily small, in particular, $\varepsilon_{j}<\frac{1}{10} \delta_{j}$. Hence $\bigcup_{a \in \xi_{j}} B\left(a, \varepsilon_{j}\right) \subset R_{j}$ and, consequently,

$$
\left[\left|p_{j}\right|>\varepsilon\right] \cap\left[\left|p_{s}\right|>\varepsilon\right]=\varnothing \quad \text { for } s=1, \ldots, j-1 \text {. }
$$

Consider now a sequence $\left\{g_{j}\right\}_{j=1}^{n}$ s. t. $g_{j} \in \mathscr{P}_{N_{j}}$ and $\left\|p_{j}-g_{j}\right\|_{\infty}<\frac{1}{10}$ for $j=1, \ldots, n$. We prove a minorization of $\left\|\sum\left|g_{j}\right|\right\|_{\infty}$ using a simple reasoning of successive perturbations based on Lemma 4.

Proceeding by induction, we define inductively points $x_{j} \in S$ so that $x_{j} \in \xi_{j+1}$ and $\left|q_{s}\left(x_{j}\right)\right|>c \varepsilon$ for $s=1, \ldots, j$. Let us describe the inductive step. Since $x_{j} \in \xi_{j+1}$, we have $p_{j+1}\left(x_{j}\right) \approx 1$, hence $\left|q_{j+1}\left(x_{j}\right)\right|>\frac{4}{5}$. Define

$$
h(\zeta)=\left\langle\zeta, x_{j}\right\rangle^{L} q_{j+1}(\zeta) \quad(L \text { to be specified })
$$

which is of degree $L+N_{j+1}$. Since $\left|h\left(x_{j}\right)\right|>\frac{4}{5}$ still, Lemma 4 yields a point $x \in S$ satisfying $d\left(x_{j}, x\right) \geqslant \varepsilon_{j+1}$ and $|h(x)|>c\left(1-\varepsilon_{j+1}\right)^{N_{j+1}+L}$. Thus if $L=o\left(N_{j+1}\right)$, we get, by definition of $\varepsilon_{j+1}$,

$$
\left|q_{j+1}(x)\right| \geqslant|h(x)| \geqslant c^{\prime} \varepsilon .
$$

Since also $\left.\left\langle x, x_{j}\right\rangle\right|^{L}>c^{\prime} \varepsilon$, it will follow that $d\left(x, x_{j}\right)=o\left(\delta_{j+1}\right)$ provided $L$ is sufficiently large (compatible with $L=o\left(N_{j+1}\right)$ ). Again, by construction, since $B\left(x_{j}, \frac{1}{10} \delta_{j+1}\right) \subset R_{j+1}$, we get $x \in R_{j+1}$. Moreover, $x \notin B\left(x_{j}, \varepsilon_{j+1}\right)$ and, since the elements of $\xi_{j+1}$ are $\delta_{j+1}$-separated and $d\left(x, x_{j}\right)=o\left(\delta_{j+1}\right)$, also $x \notin$ $\bigcup_{a \in \xi_{j+1}} B\left(a, \varepsilon_{j+1}\right)$. Hence, $x \in R_{j+2}$ and $x_{j+1} \in \xi_{j+2}$ can be chosen with $d\left(x, x_{j+1}\right)$ $<2 \delta_{j+2}$. The fact that $\delta_{j+2}$ is small enough with respect to $N_{j+1}=d\left(q_{j+1}\right)$ implies $\left|q_{j+1}\left(x_{j+1}\right)\right| \geqslant c^{\prime} \varepsilon / 2$. Further, $d\left(x_{j}, x_{j+1}\right)=o\left(\delta_{j+1}\right)$, and the inequalities $\left|q_{s}\left(x_{j+1}\right)\right|$ $>c \varepsilon, 1 \leqslant s \leqslant j$, are therefore essentially preserved. This completes the argument.

\section{REFERENCES}

1. J. Bourgain, Bilinear forms on $H^{\infty}$ and bounded bianalytic functions, Trans. Amer. Math. Soc. (to appear).

2. A. Pelczyński, Banach spaces of analytic functions and absolutely summing operators, CBMS Regional Conf. Ser. in Math., No. 30, Amer. Math. Soc., Providence, R. I., 1977.

3. W. Rudin, Function theory in the unit ball of $C^{N}$, Springer-Verlag, New York, 1980.

4. Y. Katznelson, Introduction to harmonic analysis, Wiley, New York, 1968.

Department of Mathematics, Vruje Universiteit Brussel, Pleinlaan 2, F7, 1050 Brussels, BELGIUM 